Schweizerisches Jahrbuch für Entwicklungspolitik

$20 \mid 2001$

Privatsektorförderung im Rahmen der Entwicklungszusammenarbeit

\title{
1.Internationale Finanzbeziehungen
}

\section{Bruno Gurtner}

\section{(2) OpenEdition \\ Journals}

Electronic version

URL: http://journals.openedition.org/sjep/839

DOI: $10.4000 /$ sjep.839

ISSN: 1663-9677

Publisher

Institut de hautes études internationales et du développement

\section{Printed version}

Date of publication: 1 avril 2001

Number of pages: 163-186

ISSN: 1660-5926

\section{Electronic reference}

Bruno Gurtner, «1.Internationale Finanzbeziehungen », Schweizerisches Jahrbuch für

Entwicklungspolitik [Online], 20 | 2001, Online erschienen am: 03 September 2012, abgerufen am 08 September 2020. URL : http://journals.openedition.org/sjep/839 ; DOI : https://doi.org/10.4000/sjep. 839 


\section{INTERNATIONALE FINANZBEZIEHUNGEN*}

D AS JAHR 2000 war geprägt durch Verbesserungen der wirtschaftlichen und finanziellen Lage der Entwicklungs- und Transitionsländer sowie durch anhaltende Reformdiskussionen. Von der wirtschaftlichen Erholung profitierten allerdings nicht alle Länder und Regionen im gleichen Ausmass. Auch die Reformbemühungen für die internationale Finanzordnung und die Bretton-Woods-Institutionen blieben in wichtigen Bereichen hinter den Erwartungen zurück. Der Internationale Währungsfonds und die Weltbank sahen sich - gleich anderer multilateralen Organisationen - einer intensivierten Globalisierungskritik ausgesetzt. Die wachsende Ungleichheit und die anhaltenden Armutsprobleme wurden. jedoch als dringend zu lösende globale Probleme ersten Ranges anerkannt. Qualität und Geschwindigkeit der Entschuldung der ärmsten Länder wurden gesteigert, doch die Finanzierung bleibt ungesichert.

\subsection{FINANZFLÜSSE IN ENTWICKLUNGS- UND TRANSITIONSLÄNDER}

Der Nettoressourcenzufluss in die Entwicklungs- und Transitionsländer hatte 1999 einen Tiefpunkt erreicht. Für das Jahr 2000 blieben die Zahlen und deren Beurteilungen widersprüchlich. Seitens der privaten Finanzmärkte sprach man von Normalisierung, während der IWF von schwierigen Konditionen schreibt. Steigerungen verzeichneten direkte Auslandsinvestitionen und - wie schon im Vorjahr - die öffentliche Entwicklungshilfe.

Die OECD wies für 1999 erneut einen Rückgang der gesamten (prịaten und öffentlichen) Finanzflüsse in Entwicklungs- und Transitionsländer aus, nämlich von 239,8 (1998) auf 193,5 Milliarden Dollar' (vgl. Tabelle 1). Ein gleiches Bild gab die Weltbank in ihrem Bericht Global Development Finance 2000 wieder ${ }^{2}$. Danach sanken die langfristigen Nettoflüsse in Entwicklungsländer auf 290,7 Milliarden Dollar (Vorjahr 318,3 Mrd. \$). Für das Jahr 2000 erwartet die Weltbank eine bescheidene Erhöhung. Auch die Zahlen des IWF bestätigten diese Entwicklung ${ }^{3}$.

$\square$ JSDW 1997, zu den unterschiedlichen Datenerhebungen in den verschiedenen internationalen Organisationen, Kasten S. 13/14.

\section{$\square$ Erneut leichte Steigerung der öffentlichen Entwicklungshilfe}

Die öffentliche Entwicklungshilfe ihrer Mitgliedsländer stieg gemäss provisorischen Angaben der OECD zum zweiten Mal hintereinander auf nunmehr 54,0 Milliarden Dollar. Die OECD führt dies einerseits auf besondere Leistungen Japans an die von der Finanzkrise betroffenen asiatischen Länder und anderseits auf die inter-

* Bruno Gurtner: Ökonom, Bern, bei der Arbeitsgemeinschaft Swissaid/Fastenopfer/Brot für alle/Helvetas/Caritas, zuständig für den Bereich Internationale Finanzbeziehungen.

1. OECD, News Release (DAC High Level Meeting). Paris, 12. Mai 2000.

2. The World Bank, Global Development Finance 2000 (GDF), Vol. I, Tabelle 2.1, S. 36.

3. IMF, International Capital Markets. Developments, Prospects and Key Policy Issues. Washington, September 2000, Tabelle 3.2, S. 46/47. 
nationale Hilfe an die Flüchtlinge aus Kosovo zurück. Damit lagen die OECDLänder aber immer noch zwölf Prozent unter dem 1992 geleisteten Beitrag.

Tabelle 1: Nettokapitalströme in die Entwicklungs- und Transitionsländer (in Mrd. \$ zu laufenden Preisen)

\begin{tabular}{lrrr}
\hline & $\mathbf{1 9 9 7}$ & $\mathbf{1 9 9 8}$ & $\mathbf{1 9 9 9}^{\text {p }}$ \\
\hline I. Öffentliche Entwicklungsfinanzierung & $\mathbf{7 5 . 3}$ & $\mathbf{8 8 . 3}$ & $\mathbf{8 0 . 0}$ \\
\hline 1. Staatliche Entwicklungshilfe ${ }^{*}$ & 47.7 & 49.7 & 54.0 \\
\hline davon: Bilateral & 32.4 & 35.1 & 37.0 \\
\hline Multilateral & 15.3 & 17.0 & 10.6 \\
\hline 2. Öffentliche Hilfe ** & 5.6 & 7.0 & 6.5 \\
\hline davon: Bilateral & 4.0 & 4.5 & 4.0 \\
\hline Multilateral & 1.6 & 2.5 & 2.5 \\
\hline 3. Andere öffentliche Mittel ${ }^{* *}$ & 22.0 & 31.7 & 19.5 \\
\hline davon: Bilateral & 5.9 & 12.8 & 15.0 \\
\hline Multilateral & 16.0 & 18.9 & 4.5 \\
\hline II. Exportkredite & $\mathbf{4 . 8}$ & $\mathbf{4 . 0}$ & $\mathbf{5 . 0}$ \\
\hline davon: Kurzfristig & 0.6 & 0.5 & 0.6 \\
\hline III. Private Finanzströme & $\mathbf{2 3 9 . 7}$ & $\mathbf{1 4 7 . 5}$ & $\mathbf{1 0 8 . 5}$ \\
\hline 1. Direktinvestitionen & 102.3 & 118.0 & 98.0 \\
\hline 2. Internationale Bankausleihen & 12.0 & -65.0 & -64.0 \\
\hline davon: Kurzfristig & 12.0 & -70.0 & -54.0 \\
\hline 3. Bondanleihen & 83.2 & 39.8 & 39.0 \\
\hline 4. Andere (einschliesslich Portfolio) & 37.0 & 49.1 & 30.0 \\
\hline 5. Leistungen von NROs & 5.2 & 5.6 & 5.5 \\
\hline Total Netto-Zuflüsse & $\mathbf{3 1 9 . 7}$ & $\mathbf{2 3 9 . 8}$ & $\mathbf{1 9 3 . 5}$ \\
\hline
\end{tabular}

Quelle: OECD News Release, 12 Mai 2000.

* Zuschüsse und Darlehen an Entwicklungsländer.

** Leistungen an die Übergangsländer.

***Nichtkonzessionäre Mittel.

${ }^{\mathrm{P}}$ provisorische Angaben.

Die G-7-Länder leisteten bloss 0,21 Prozent ihres Bruttosozialproduktes, während die übrigen OECD-Länder 0,44 Prozent für die internationale Zusammenarbeit verwendeten. Zehn Länder zahlten im Vergleich zum Vorjahr weniger, sieben erhöhten ihre Leistungen.

Die OECD wies die Schweizer Hilfe mit einem Betrag von 976 Millionen Dollar aus (Vorjahr 898 Mio. \$). Dies entsprach einem Anteil von 0,35 Prozent am Bruttosozialprodukt (Vorjahr 0,32\%). Ein Teil dieser Mehrleistungen ist darauf zurückzuführen, dass bisher nicht eingeschlossene Leistungen neu in der Statistik mitgezählt werden.

[D] JSDW 2001, Kapitel 7, EZA Zusammenarbeit mit den Entwicklungsländern

Die OECD nahm die voraussichtlich im ersten Quartal des Jahres 2002 durchzuführende UNO-Konferenz über die Finanzierung der Entwicklung zum Anlass, eine verstärkte Kohärenz zwischen den privaten und öffentlichen Kapitalflüssen $\mathrm{zu}$ postulieren. Die öffentlichen Leistungen müssten sich an die sich verstärkende Globalisierung und an die zunehmende Bedeutung privater Finanzflüsse anpassen. Ihr Fokus müsse klar auf Strategien zur Armutsbekämpfung und zur

4. OECD, The DAC Journal, Development Cooperation 1999 Report, OECD Paris 2000. 
Integration der Entwicklungsländer in die Weltwirtschaft ausgerichtet sein 4 . Punkte dieser Agenda sollen demnach u.a. sein : Internationale Finanzarchitektur, Überprüfung des Finanzsektors, «Corporate Governance», Korruptionsbekämpfung, Förderung der Direktinvestitionen, Partnerschaft mit der Privatwirtschaft, diszipliniertere Exportkredittätigkeit. Erwähnt wird dabei auch das Problem der gebundenen Hilfe. Ein dazu im Juni verabschiedeter Beschluss der OECD sieht allerdings zahlreiche Ausnahmen vor.

Während die öffentliche Entwicklungshilfe stieg, sanken die übrigen (nicht-konzessionären) öffentlichen Leistungen (vgl. Tabelle 1, Punkt 3). Dies ist auf die Rückzahlung und die geringere Neubeanspruchung von Überbrückungskrediten im Gefolge der Finanzkrise zurückzuführen.

Die Weltbank nahm erfreut Kenntnis von der leichten Steigerung der öffentlichen Hilfe und untersuchte die Gründe, die zum Rückgang im letzten Jahrzehnt geführt haben ${ }^{5}$. Wie im Vorjahr legte die Bank in ihren Ausführungen ein grosses Gewicht auf die Qualität und die effiziente Allokation.

\section{$\square$ Der Rückgang der privaten Kapitalflüsse hielt an}

Die OECD stellte für das Jahr 1999 gemäss provisorischen Zahlen weiterhin einen Rückgang der gesamten privaten Kapitalflüsse auf noch 108,5 Milliarden Dollar fest (Vorjahr 147,5 Mrd. \$, vgl. Tabelle 1). Die Weltbank bestätigte diesen Trend. Ihre Statistiken wiesen einen Rückgang von 267,7 auf 238,7 Milliarden Dollar aus ${ }^{6}$. Für den IWF haben sich die privaten Flüsse auf tiefem Niveau stabilisiert. 1999 beliefen sich gemäss IWF die privaten Kapitalflüsse noch gerade auf ein Drittel des Höchststandes von 19957. Das Institute of International Finance $(I I F)$, ein Think Tank der grössten privaten Banken, bestätigte dies und sagte für das Jahr 2000 sogar eine deutliche Steigerung der privaten Kapitalflüsse voraus, die auch im Jahr 2001 anhalten werde ${ }^{8}$.

Mit diesem Rückgang zu einer Stabilisierung auf tiefem Niveau hat sich auch die Zusammensetzung der privaten Kapitalströme deutlich verändert.

Ca JSDW 1998, zu den unterschiedlichen Formen privater Kapitalflüsse vgl. S. 140, Kasten «Private Kapitalflüsse».

Die direkten Auslandsinvestitionen wuchsen auch während der Krise stetig an und machen nun den Hauptanteil der privaten Kapitalflüsse aus. Damit ist die relative Stabilität der direkten Auslandsinvestitionen bestätigt. Für den IWF hängt dies zumindest damit zusammen, dass ein grosser Teil der erzielten Gewinne im gleichen Land reinvestiert wird. Für 1999 wies die OECD allerdings einen Rückgang um einen Sechstel aus (vgl. Tab. 1). Auch das IIF erwartet für das Jahr 2000 ebenso wie für 2001 einen leichten Rückgang der direkten Auslandsinvestitionen.

Portfolio-Investitionen hatten im Jahre 1998 offenbar einen Tiefpunkt erreicht. 1999 waren sie wieder leicht ansteigend, wie die entsprechenden Zahlen von IWF, Weltbank und IIF nachweisen. Portfolio-Investitionen flossen vor allem auf

5. GDF 2000, aaO. Chapter 3: Trends in Official Finance, S. $57 \mathrm{ff}$.

6. GDF 2000, aaO. Tabelle 2.1, S. 36.

7. IMF, International Capital Markets, aaO. Tabelle 3.2, S. 46/47.

8. Institute of International Finance, Capital Flows to Emerging Market Economies, Washington, 23. September 2000 (www.iif.com). 
die Börsen der asiatischen Schwellenländer, nach Südafrika, Mexiko und Brasilien. Portfolio-Investitionen schwanken jedoch deutlich, je nach Ertragsaussichten an den Börsen. So führten die Turbulenzen auf den führenden Börsen der Welt anfangs 2000 auch zu einer Verminderung der Portfolio-Investitionen in zahlreichen Schwellenländern ${ }^{9}$.

Hingegen hielt 1999 gemäss allen verfügbaren Statistiken der Rückgang der Bankausleihen und der Obligationen (Bonds) weiter an. Auch für das Jahr 2000 war noch kein markanter Trendwechsel festzustellen. Deutliche Unterschiede waren allerdings zwischen den einzelnen betroffenen Ländern sichtbar. Anleger bevorzugten nach dem Debakel der Finanzkrisen sicherere Anlagen. Einzelne Länder, die sich von der Finanzkrise erholten, und deren Politik von den internationalen Märkten als gut erachtet wurden, erhielten wieder Zugang zu neuen Mitteln, auch zu günstigeren Bedingungen ${ }^{10}$. Einzelne Länder tilgten in grossem Ausmass Kredite zurück.

Obwohl die Finanzmarktaktivitäten in den fortgeschrittenen Ländern zur Normalität zurückgekehrt seien, so der IWF, bleibe die externe Finanzierung für viele aufstrebenden Märkte ungewöhnlich begrenzt. Die Zinsaufschläge auf die externen Schuldeninstrumente dieser Länder seien nach wie vor sehr hoch. Die international tätigen Banken würden ihren Rückzug aus den aufstrebenden Märkten fortsetzen. Dies führe zu einer weiterhin hohen Volatilität und zu fortdauernden erheblichen Anfälligkeiten ${ }^{11}$.

Die UNCTAD sieht in den globalen Ungleichgewichten (harte oder weiche Landung in den USA? Schwäche des Euro, Japans Dauerrezession) eine wichtige Ursache für die Unsicherheiten auf den Finanzmärkten ${ }^{12}$, die sich auch für die Entwicklungsländer negativ auswirkten.

Weitere Unsicherheitsfaktoren auf den internationalen Kapitalmärkten sind die Preissteigerungen beim Erdöl und die rückläufigen Defizite oder gar Überschüsse in den öffentlichen Haushalten zahlreicher Industrieländer. Ende 2000 schienen die gesteigerten Einnahmen der Erdöl produzierenden Länder noch keinen sichtbaren Niederschlag in Höhe und Zusammensetzung der internationalen Kapitalflüsse gezeitigt zu haben. Überschüsse (oder geringere Defizite) der öffentlichen Haushalte haben nicht nur eine reduzierte Nachfrage nach Finanzmitteln zur Folge. Vielmehr ist damit auch eine Reduktion des Angebotes an sicheren Geldanlagen verbunden. Diese Entwicklung steht dem Trend nach Anlagen in sichereren Geldinstrumenten entgegen und erhöht ihrerseits die Unsicherheiten den Märkten.

In ihrem Bericht über die am wenigsten entwickelten Länder (LDC) für das Jahr 2000 beklagt die UNCTAD, dass diese Länder im letzten Jahrzehnt einen Rückgang ihrer nominellen Nettokapitalzuflüsse um einen Viertel hinnehmen mussten. Der Bericht untersucht zudem nationale und internationale Hindernisse für den Zustrom privaten Kapitals in die LDC13.

9. UNO, Financial Flows to Developing and Transition Economies and Main Policy Developments. An Excerpt from World Economic and Social Survey, 2000.

10. Bank für internationalen Zahlungsausgleich (BIZ), 70. Jahresbericht, Basel 2000.

11. IMF, Die Vorteile der Weltwirtschaft für alle sichern. Jahresbericht 2000, Washington 2000, S. 25.

12. UNCTAD, Trade and Development Report 2000, Chapter III, New York und Genf 2000.

13. UNCTAD, The Least Developed Countries 2000 Report, Aid, Private Capital Flows and External Debt: The Challenge of Financing Development in LDCs, New York und Genf 2000. 


\section{$\square$ Die Verstärkung der internationalen Finanzordnung}

Einige Länder (Südkorea, Thailand, Malaysia und Brasilien) haben sich erstaunlich rasch von der Finanzkrise der letzten Jahre erholt, zumindest bezogen auf ihre makroökonomischen Fundamentaldaten. Mehr Zeit braucht es offensichtlich für die Korrektur der verheerenden, negativen sozialen Auswirkungen. Auch strukturelle Änderungen, z.B. im Finanz- und Unternehmungssektor, wurden zum Teil nur zögerlich angegangen ${ }^{14}$. Es machte sich eine gewisse Reformmüdigkeit breit.

JSDW 2000, S. 127: Auswirkungen der Finanzkrise.

Die Finanzkrisen der 90er Jahre haben die Schwächen im internationalen Finanzsystem aufgedeckt, haben verdeutlicht, dass die Globalisierung neben Vorteilen auch erhebliche Risiken mit sich bringt. Eine Reform der internationalen Finanzarchitektur war angesagt. Zwar sind bis Ende des Jahres 2000 zahlreiche Verbesserungen durchgeführt worden. Doch entscheidende Reformelemente sind politisch blockiert ${ }^{15}$.

Fortschritte erzielten IWF, die Weltbank, das Forum für Finanzstabilität (FSF), die Bank für Internationalen Zahlungsausgleich (BIZ) und deren verschiedene Arbeitsgruppen, die OECD, die G-20 und weitere internationale Instanzen ${ }^{16}$ zum Beispiel in der besseren und zeitgerechteren Bereitstellung von Informationen (Transparenz) für die Märkte und für die Öffentlichkeit und in der Entwicklung von Standards, Verhaltensgrundsätzen und Richtlinien ${ }^{17}$.

[D JSDW 2001, zu den innerinstitutionellen Reformen bei IWF und Weltbank, vgl. die unten stehenden Abschnitte 1.4.1. und 1.4.2.

Zur Stärkung der Finanzsysteme und zur frühzeitigen Erkennung von drohenden Krisen sollen die Banken- und Finanzsektoren der einzelnen Länder gestärkt werden. Ein wichtiges Element bildet dabei das gemeinsame Programm von IWF und Weltbank zur Überprüfung des Finanzsektors (Financial Sector Assessment Program, FSAP). Das Pilotprogramm umfasst 12 Länder, ausser Kanada alles Länder mit aufstrebenden Märkten. An der Jahresversammlung von IWF und Weltbank in Prag im September 2000 erklärte Bundesrat Kaspar Villiger, die Schweiz sei ebenfalls bereit, sich diesem Überprüfungsprogramm zu unterziehen $^{18}$. Diese Offenheit ist nach Villiger im Zusammenhang mit den Kritiken am Finanzplatz Schweiz zu sehen: «Wir haben nichts zu verstecken».

Der Früherkennung von Krisen soll auch eine Erarbeitung von verschuldungsund reservenbezogenen Indikatoren externer Anfälligkeit dienen. Fortgeführt

14. BIZ, 70. Jahresbericht 2000, aaO., S. 39ff.

15. Gurtner Bruno, «Geld denkt nicht. Die Verantwortlichen der internationalen Finanzmärkte haben ihre Versprechen nicht eingehalten. »Cash, 29. September 2000.

16. Eine gute Übersicht über diese verschiedensten Institutionen bietet ein IMF Fact Sheet: A Brief Guide to Committees and Groups, 17. Juli 2000 (www.imf.org/external/np/exr/facts/groups.htm).

17. IMF, Jahresbericht 2000, aaO., S. $41 \mathrm{ff}$., sowie zahlreiche im Internet publizierte Dokumente (www.imf.org).

18. Villiger Kaspar, Bundesrat, Statement an der Pressekonferenz in Anschluss an die Tagung der Zehnergruppe und des Internationalen Währungs- und Finanzausschusses. Jahrestagung der Bretton-WoodsInstitutionen 2000 (Vervielfältigung EFV, ohne Datum). Zum FSAP vgl. auch: IMF, Jahresbericht 2000, aaO. S. 45 sowie: IMF, Progress Report on the Bank-Fund Financial Sector Liaison Committee, 29. März 2000 (www.imf.org/external/np/mae/fslc/032900.htm). 
wurde die Debatte um ein angemessenes Wechselkurssystem ${ }^{19}$ sowie die Diskussion um Kapitalverkehrsliberalisierung und Kapitalverkehrskontrolle. Dem früheren Übergewicht der Liberalisierungsidee steht heute eine grössere Bereitschaft gegenüber, in bestimmten Fällen den Nutzen von Kontrollen zu akzeptieren. Kontrollen ersetzen jedoch nicht eine gute Wirtschaftspolitik ${ }^{20}$.

Wenig Fortschritte gab es in der Frage des Einbezuges des privaten Sektors in die Vermeidung und Überwindung von Krisen zu verzeichnen. Zwar sind zahlreiche Papiere verfasst worden, und alle Kreise betonen die Wichtigkeit des Anliegens. Doch hinsichtlich konkreter Massnahmen blockieren sich unterschiedliche Auffassungen. Während europäische Länder einschliesslich der Schweiz gewisse allgemeingültige Regeln begrüssen würden, lehnen die USA und der private Bankensektor solche ab. Sie ziehen Von-Fall-zu-Fall-Lösungen vor. Keines der andiskutierten Instrumente ist verwirklicht, weder ein Rahmen für die Mitfinanzierung von IWF-unterstützten Krisenprogrammen noch Regeln für SchuldnerGläubiger-Verhandlungen, noch Modalitäten für einen temporären Zahlungsstopp oder für die Gleichstellung von Bonds-Gläubigern mit Banken-Gläubigern $^{21}$. Die Eidg. Finanzverwaltung hat zur Frage des Einbezuges des privaten Sektors eine Studie in Auftrag gegeben ${ }^{22}$.

Der IWF widmete der Reform der globalen Finanzarchitektur in seinem Jahresbericht ein eigenes Kapital und legte auch sonst periodisch interne Fortschrittsberichte offen ${ }^{23}$. Dazu sind auch zahlreiche kritische Publikationen erschienen, grösstenteils in englischer Sprache ${ }^{24}$. Der neue IWF-Direktor Horst Köhler setzte eine aus Vertretern internationaler Banken bestückte Capital Markets Consultative Group ein, die ein strukturierter Dialog mit den Grossbanken führen soll.

Die Schweiz hatte ihre Position zu den einzelnen Fragen bereits 1999 in zwei Papieren publiziert und passte sie im Laufe des Jahres 2000 bei einzelnen Fragen den Diskussionen an ${ }^{25}$.

[D JSDW 2000, zu den bundesrätlichen Positionen für die internationale Finanzordnung, vgl. S. 129.

In der Frühjahrssession der eidg. Räte reichten acht Mitglieder des Parlamentes in beiden Räten sechs gegenseitig unterstützte Vorstösse zu einzelnen Fragen der

19. IMF, Exchange Rate Regimes in an Increasingly Integrated World Economy, Issue Briefs for 2000 , Juni 2000 (www.imf.org/external/np/exr/ib/2000/062600.HTM).

20. Vgl. dazu IMF, Capital Controls: Country Experiences with their Use and Liberalization. Occasional Paper 190, Washington 2000 (oder advance copy: www.imf.org/external/pubs/ft/capcon/index/htm)

21. IMF, Involving the Private Sector in the Resolution of Financial Crisis - Standstills - Preliminary Considerations. 5. September 2000 (www.imf.org/external/np/pdr/sstill/2000/eng).

22. Barry Eichengreen, Can the Moral Hazard caused by IMF-bailouts be reduced?, ICBM/CEPR, Genf/London 2000.

23. IMF, Jahresbericht 2000, aaO. Kapitel 4, S. 39ff., sowie IMF, Report of the Managing Director to the International Monetary and Financial Committee (IMFC) on Progress in Strengthening the Architecture of the International Financial System and Reform of the IMF, Washington, 19. September 2000 (www.imf.org/

24. Eine gute Übersicht in deutscher Sprache mit reicher Bibliographie hat WEED publiziert: WEED, Spekulieren, Regulieren. Kapital braucht Kontrolle. Die internationalen Finanzmärkte: Funktionsweise - Hintergründe - Alternativen, Bonn, August 2000 (in der Schweiz beziehbar bei der Arbeitsgemeinschaft der Hilfswerke, Tel. 03139093 37).

25. IMF, Executive Director for Azerbaijan, Kyrgyz Republic, Poland, Switzerland, Tajikistan, Turkmenistan, Uzbekistan, Activity Report 2000, S. 9ff., sowie: Reformen im IWF: Diskussion beginnt erst. Info plus, Newsletter des EFD, Nr. 9, März 2000. 
internationalen Finanzordnung ein und stellten sie an einer Medienkonferenz am 23. März 2000 der Öffentlichkeit vor ${ }^{26}$. Eine Motion Eymann (NR, lib, BS) zur Schaffung von Schiedsverfahren zum Interessenausgleich zwischen Schuldnerländern und Gläubigern wurde als Postulat überwiesen, ebenso ein Postulat Strahm (NR, SP, BE) Privater Sektor und Einbezug in die Finanzmarktrisiken. Teile eines gleich lautenden Postulates Langenberger (StR, FDP, VD) und Pelli (NR, FDP, TI) über Transparenz im IWF wurden ebenfalls überwiesen. Die Überweisung des Postulates Gysin (NR, SP, BS) zur Reform des Stimmrechtes im IWF wurde knapp nicht überwiesen. Zum Paket dieser Vorstösse gehörte im Weitern eine gleich lautende Interpellation David (StR, CVP, SG) und Baumann (NR, G, BE) über die Globalisierung im Kapitalverkehr sowie eine Frage Neirynck (NR, CVP, VD) über Transaktionen der ukrainischen Zentralbank mit Hilfe der Credit Suisse First Boston zur Täuschung des IWF. Die Arbeitsgemeinschaft der Hilfswerke hatte im Januar eine Reregulierung der internationalen Finanzmärkte gefordert ${ }^{27}$.

\section{$\square$ Der Finanzplatz Schweiz unter Druck}

Die Deregulierung der globalen Finanzmärkte hat nicht nur eine erhöhte Krisenanfälligkeit zur Folge. Vielmehr sind auch die Möglichkeiten zur Geldwäsche krimineller Gelder und zur Steuerhinterziehung drastisch gewachsen. In den letzten Jahren sind auf internationaler Ebene bedeutsame Anstrengungen unternommen worden zur Bekämpfung von Geldwäscherei und Steuerhinterziehung. Zwar sind sog. Offshore-Zentren anvisiert, aber die Schweiz ist davon ebenfalls stark betroffen. Mehrere internationale Organisationen haben sich mit diesen Fragen beschäftigt ${ }^{28}$.

Dabei ist das Schweizer Bankgeheimnis insbesondere unter Druck, einerseits durch die Publikation eines Berichtes des OECD-Fiskalausschusses über den verbesserten Zugang zu Bankinformationen zu Steuerzwecken29, anderseits durch die Bestrebungen der EU über die Besteuerung von Kapitalerträgen ${ }^{30}$. Schliesslich hatte die Arbeitsgruppe Offshore Financial Centres des Financial Stability Forum $(F S F)$ im Mai 2000 einen Bericht veröffentlicht und darin drei Kategorien von «Jurisdiktion» mit Off-shore-Aktivitäten aufgelistet ${ }^{31}$. Sie führte die Schweiz in der Gruppe I auf, jener Kategorie, deren legale Infrastruktur, deren Überwachungspraktiken und deren Kooperationswilligkeit von guter Qualität sei. Die Schweiz protestierte selbst gegen diese Kategorisierung.

26. Reform der internationalen Finanzordnung. Medienkonferenz vom 23. März 2000 (Vervielfältigung, erhältlich beim Autor dieser Übersicht), sowie: Amtliches Bulletin, Ständerat, Sommersession 2000, Nationalrat, Herbstsession 2000, Bern 2000 (vgl. auch www.parlament.ch/)).

27. Swiss Coalition News, Nr. 22, Januar 2000, Bern.

28. Vgl. dazu: Jacques de Watteville, Das Bankgeheimnis. Das Schweizer Bankgeheimnis angesichts der jüngsten internationalen Entwicklungen, namentlich in der OECD und der EU, in: Die Volkswirtschaft - Magazin für WirtschaftsPolitik, 7/2000, Bern.

29. OECD, Improving Access to Bank Information for Tax Purposes, OECD, ISBN 92-64-17649-7.

30. Vgl. die Diskussion zur Interpellation Schlüer im NR, Herbstsession 2000. Bern 2000 (vgl. auch www.parlament.ch).

31. BIS Press Release, Financial Stability Forum, 26. Mai 2000 (www.bis.org/press/p00526.htm). 


\subsection{AUSSENVERSCHULDUNG}

Die Schulden der Entwicklungsländer stiegen auch 1999 weiter. Die HIPCInitiative wurde erweitert und beschleunigt. Die künftig von den Ländern in partizipativen Verfahren zu erarbeitenden Armutsstrategiepapieren (Poverty Reduction Strategy Paper, PRSP) werden künftig die Grundlage für die multilaterale und wohl auch für die bilaterale öffentliche Entwicklungsfinanzierung bilden. NRO plädierten insbesondere für die Schaffung internationaler Insolvenzmechanismen.

\section{$\square$ Trends}

Die gesamten Schulden aller Entwicklungs- und Transitionsländer erhöhten sich gemäss Weltbank leicht auf 2554,0 Milliarden Dollar ${ }^{32}$. Davon sind 2070,7 Milliarden langfristige Schulden (vgl. Tabelle 2). Die Schuldenindikatoren entwickelten sich regional und ländergruppenspezifisch uneinheitlich (Tabelle 3).

Tabelle 2: Die Gesamtverschuldung der Entwicklungs- und Transitionsländer (in Mrd. \$)

\begin{tabular}{lrrrr}
\hline & $\mathbf{1 9 8 0}$ & $\mathbf{1 9 9 0}$ & $\mathbf{1 9 9 8}$ & $\mathbf{1 9 9 9}$ \\
\hline Langfristige Verschuldung & 452 & 1181 & 2030 & 2070 \\
\hline - Öffentlich und öffentlich garantiert & 381 & 1116 & 1529 & 1580 \\
\hline Offizielle Kreditoren & 178 & 605 & 853 & 876 \\
\hline Multilateral & 49 & 208 & 326 & 346 \\
\hline Bilateral & 129 & 397 & 527 & 530 \\
\hline private, öffentlich garantiert & 203 & 511 & 676 & 705 \\
\hline - private nicht garantiert & 71 & 66 & 501 & 491 \\
\hline - IWF Kredite & 12 & 35 & 94 & 81 \\
\hline Kurzfristige Kredite & 146 & 245 & 412 & 402 \\
\hline Gesamtverschuldung & $\mathbf{6 0 9}$ & $\mathbf{1 4 6 0}$ & $\mathbf{2 5 3 6}$ & $\mathbf{2 5 5 4}$ \\
\hline
\end{tabular}

Quelle: The World Bank, Global Development Finance 2000, S. 238/239.

Tabelle 3: Schuldenindikatoren 1990, 1998 und 1999

\begin{tabular}{lrrrrrr}
\hline $\begin{array}{l}\text { Ländergruppe } \\
\text { (in Prozenten) }\end{array}$ & $\begin{array}{r}\text { D/EX* } \\
\mathbf{1 9 9 0}\end{array}$ & $\begin{array}{r}\text { D/EX } \\
\mathbf{1 9 9 8}\end{array}$ & $\begin{array}{r}\text { D/EX } \\
\mathbf{1 9 9 9}\end{array}$ & $\begin{array}{r}\text { DS/EX* } \\
\mathbf{1 9 9 0}\end{array}$ & $\begin{array}{r}\text { DS/EX } \\
\mathbf{1 9 9 8}\end{array}$ & $\begin{array}{r}\text { DS/EX } \\
\mathbf{1 9 9 9}\end{array}$ \\
\hline Alle Länder & 162.5 & 147.9 & 136.6 & 7.8 & 7.0 & 7.2 \\
\hline Ostasien/Pazifik & 108.4 & 104.9 & 94.4 & 15.7 & 13.3 & 14.8 \\
\hline Europa/Zentralasien & - & 133.8 & 133.7 & - & 14.7 & 14.8 \\
\hline Lateinamerika/Karibik & 255.7 & 210.5 & 194.6 & 12.3 & 12.4 & 13.1 \\
\hline Mittlerer Osten/Nordafrika & 113.9 & 129.1 & 111.3 & 15.1 & 14.0 & 11.0 \\
\hline Südasien & 327.4 & 189.1 & 162.5 & 29.0 & 18.9 & 14.5 \\
\hline Afrika südl. Sahara & 209.6 & 238.9 & 225.1 & 12.9 & 14.7 & 14.8 \\
\hline Länder mit tiefem Einkommen & 257.8 & 168.9 & 152.0 & 10.2 & 6.0 & 5.7 \\
\hline Länder mit mittlerem Einkommen & 137.2 & 140.9 & 128.5 & 7.2 & 7.3 & 7.6 \\
\hline
\end{tabular}

Quelle: WDR 2000, S. 238ff.

* $D / E X=$ Verhältnis Schulden/Exporte von Gütern und Dienstleistungen; DS/EX = Verhältnis Schuldendienst/ Exporte von Gütern und Dienstleistungen.

$\square$ Die Weiterentwicklung der HIPC-Initiative: Armutsstrategien

Bekanntlich hatten der G-7-Gipfel von Köln und die Jahrestagung von IWF und Weltbank 1999 beschlossen, die multilateralen Schuldeninitiative (HIPC-Initia- 
tive) $\mathrm{zu}$ beschleunigen, $\mathrm{zu}$ vertiefen, $\mathrm{zu}$ verbreitern und sie direkter mit der Armutsbekämpfung zu verbinden. Die einzelnen Länder müssen neu unter Einschluss ihrer Zivilgesellschaft und der Partner sog. Poverty Reduction Strategy Papers (PRSP) verfassen, die in Zukunft auch die Grundlagen bilden sollen für die öffentliche internationale Entwicklungsfinanzierung.

JSDW 2000, Weiterentwicklung der HIPC-Initiative, S. 131-134.

Dieses Konzept beschäftigte im Jahre 2000 die Entwicklungsdiskussion in hohem Ausmass. IWF und Weltbank legten seit dem Spätherbst 1999 ihren Exekutivräten zahlreiche Papiere zur Diskussion und Entscheidung für die Weiterentwicklung der HIPC-Initiative und der PRSP vor und veröffentlichten diese auf ihren Websites. Zu beachten sind insbesondere die Progress Reports, die sowohl das Frühjahrestreffen im April 2000 in Washington wie auch für die Jahresversammlung in Prag im September erstellt wurden ${ }^{33}$. In zahlreichen Veranstaltungen mit Regierungen und mit NRO wurden kritische Elemente der neuen Strategie erörtert. Es entstand in kurzer Zeit eine umfangreiche, kaum mehr überschaubare und ständig weiterwachsende Literatur. Dies verdeutlicht die Bedeutung, Komplexität und auch die Umstrittenheit der neuen Politik.

Die DEZA veranstaltete an ihrem Koordinatorenseminar 2000 eine Diskussion zum Thema. Auch die Arbeitsgemeinschaft der Hilfswerke führte im Mai 2000 ein internes PRSP-Seminar durch, um die Programmverantwortlichen der Hilfswerke für HIPC-Länder mit den PRSP vertraut zu machen ${ }^{34}$.

\section{Kasten: Die weltweite Auseinandersetzung um den PRS-Prozess}

$\sqsupset$ Die Ansprüche des PRSP sind enorm. Ein PRSP soll eine umfassende Strategie der Armutsbekämpfung sein, makroökonomische, strukturelle, sektorielle und soziale Elemente konsistent zusammenführen, in den Ländern selber und erst noch in partizipativer Weise mit der Zivilgesellschaft und in Koordination mit den Gebern erarbeitet werden. Das ist ein äusserst komplizierter, anforderungsreicher und zeitintensiver Prozess.

- Die Verbindung von PRSP mit der HIPC-Initiative führt zu Widersprüchen zwischen der Forderung nach rascher Entschuldung und der Forderung nach hoher Qualität des PRSP. IWF und Weltbank verlangen von den Ländern für die Qualifizierung zur HIPC-Initiative kurzfristig nur ein Interims-PRSP, während viele NRO eine klare Trennung verlangen.

• Führt dieser Prozess zu erhöhter Eigenständigkeit der Entwicklungsländer oder ist es eine neue, zusätzliche Konditionalität? Sind IWF und Weltbank bereit, ihre bisherigen Wachstumsstrategien zu erneuern und verändern?

33. Sowohl IWF wie Weltbank haben eigene HIPC/PRSP-Homepages eingerichtet: www.imf.org/external/np/hipc/, www.worldbank.org/hipc und www.worldbank.org/prsp/, wo auch Übersichten über die bisher veröffentlichten Papers aufgelistet sind. Vgl. insbesondere die letzten Progress Reports: IMF/World Bank, The Enhanced Initiative for Heavily Indebted Poor Countries. Review of Implementation, 7. September 2000 (www.worldbank.org/html/extdr/hipc/hippc090700.htm), und: IMF/ World Bank, Poverty Reduction Strategy Papers - Progress in Implementation, 7. September 2000 (www.imf.org/external/np/prsp/2000/prsp.htm).

34. Arbeitsgemeinschaft Swissaid/Fastenopfer/Brot für alle/Helvetas/Caritas, Mit «civil Society» die Armut bekämpfen? Fotoprotokoll, Seminar vom 10. Mai 2000, Bern. 
IWF und Weltbank beteuerten, bis Ende des Jahres 2000 sollten mit 20 Ländern HIPC-Vereinbarungen abgeschlossen sein. Bis Mitte Oktober erhielten elf Länder (Benin, Bolivien, Burkina Faso, Honduras, Kamerun, Mali, Mauretanien, Moçambique, Senegal, Tansania, Uganda) Schuldenerleichterungen im Ausmass von insgesamt 18, 19 Milliarden Dollar. Damit sollten diesen Ländern der Schuldendienst gemäss Angaben der Weltbank um durchschnittlich einen Drittel gesenkt werden können. Weitere neun Länder sollten bis Ende 2000 ebenfalls noch in Genuss kommen ${ }^{35}$. Letztlich sollen neu ungefähr 32 Länder im HIPCProzess zu Schuldenerleichterungen gelangen. Das wird schätzungsweise 28,6 Milliarden Dollar (Basis Ende 1999) kosten, hälftig aufzuteilen auf multilaterale einerseits und auf bilaterale und kommerzielle Gläubiger anderseits. Die Finanzierung ist noch nicht gesichert. Im Oktober 2000 billigte das US-Repräsentantenhaus eine Gesetzesvorlage über die Auslandshilfe, in der auch 435 Millionen Dollar für Entschuldungsmassnahmen vorgesehen sind. Damit wurde ein grosses Hindernis für die Finanzierung aus dem Wege geräumt.

Die Schweiz beteiligt sich insgesamt mit 160 Millionen Franken an den Kosten, 70 Millionen davon über den IWF. Die Schweiz unterstützte die Beschleunigung und den Ausbau der HIPC-Initiative, aber legte ein grosses Gewicht auf die Nachhaltigkeit des Prozesses. Notwendig seien eine gute Wirtschaftspolitik, eine klare Armutsstrategie und eine gute Regierungsführung 36 . Vorbehalte äusserte die Schweiz gegenüber der Idee, zurückfliessende Mittel aus früheren IDAKrediten künftig für die Finanzierung von Entschuldungsmassnahmen abzuzweigen, statt sie in neue Projekte zu leiten. Damit wäre die geforderte Additionalität von Entschuldungsmassnahmen nicht mehr gewährleistet.

\section{$\square$ Umschuldung öffentlicher Schulden (Pariser Klub)}

Der Pariser Klub schuldete 1999 Schulden gegenüber öffentlichen Gebern und öffentlich garantierte Schulden von sieben Ländern im Ausmass von 16 Milliarden Dollar um ${ }^{37}$. Fünf dieser Abkommen betrafen Länder mit niedrigem Einkommen (Guayana, Honduras, Moçambique, Pakistan und Sambia), Jordanien und Russland gehören zur Kategorie der Länder mit mittleren Einkommen.

Als Folge der Kosovo-Krise erhielten zudem Albanien und Mazedonien einen einjährigen Zahlungsaufschub für ihren Schuldendienst ab April 1999 zugestanden. Auch den vom Hurrikan "Mitch» betroffenen zentralamerikanischen Ländern Honduras und Nicaragua war im Dezember 1998 eine Aussetzung der bilateralen Zahlungsverpflichtungen für drei Jahre gewährt worden.

Im Jahre 2000 kamen im Pariser Klub bis Oktober 2000 Umschuldungen mit Mauretanien (102 Mio. \$), Indonesien (5400 Mio. \$), Tansania (714 Mio. \$), Sao Tomé e Principe (28 Mio. \$), Uganda (145 Mio. \$) und Ecuador (887 Mio. \$) zustande.

Die Schweiz beteiligte sich 1999 an vier Abkommen des Pariser Klubs, nämlich mit Honduras, Jordanien, Pakistan und Russland. Der Anteil der Schweiz an den

35. IMF/World Bank, HIPC-Initiative, Country Implementation Status Notes, 19. September 2000).

36. Gemäss Reden von den Bundesräten Villiger und Couchepin an der Jahresversammlung in Prag, Pressegespräche. Vgl. auch: World Bank Group, Executive Director for Azerbaijan, Kyrgyz Republic, Poland, Switzerland, Tajikistan, Turkmenistan, Uzbekistan, Report to the Governors, September 2000, S. 8.

37. The World Bank, GDF 2000, aaO., S. 71 und Appendix 3, S. $169 \mathrm{ff}$. 
Umschuldungen von 1999 beträgt insgesamt 105 Millionen Franken, wovon auf Pakistan 50,8 und auf Russland 43,5 Millionen Franken entfallen ${ }^{38}$. Bei den Umschuldungen im Pariser Klub im Jahre 2000 (bis Oktober) war die Schweiz nur im Falle von Indonesien mit ungefähr 190 Millionen Franken betroffen.

Jedes Land muss im Anschluss an die Pariser Verhandlungen selber bilaterale Abkommen mit den Schuldnerländern schliessen. Im Jahre 1999 schloss die Schweiz solche Abkommen mit Indonesien, der Zentralafrikanischen Republik und mit Bosnien-Herzegowina im Umfang von insgesamt 198 Millionen Franken $\mathrm{ab}^{39}$. Im Jahre 2000 sind bis Oktober bilaterale Abkommen mit Pakistan (51 Mio. Fr.), Honduras (3,3 Mio. Fr.) und Russland (43 Mio. Fr.) vereinbart worden. Nach dem G-7-Gipfel 1999 in Köln beschloss der Pariser Klub, HIPC-Ländern künftig die Schulden bis zu 90 Prozent zu erlassen. In schwierigsten Fällen sollten die bilateralen öffentlichen Schulden gar vollständig gestrichen werden können. Einzelne G-7-Länder kündigten inzwischen an, ihre entsprechenden Guthaben generell zu 100 Prozent zu streichen. Der Pariser Klub gewährte seither Uganda, Honduras, Benin, Burkina Faso, Mali und Senegal Entschuldungsmassnahmen im Rahmen der HIPC-Initiative. Einzig Honduras hat gegenüber der Schweiz Ausstände.

Der Pariser Klub bleibt ein geheimer Club souveräner Gläubiger. Informationen fliessen nur spärlich in Form von dürren Mitteilungen an die Medien durch das französische Finanzministerium. Internationale NRO demonstrierten im April in Paris und forderten eine bessere Transparenz und ausgewogene Schuldenverhandlungen. Auch Medien kritisierten die fehlende Transparenz ${ }^{40}$. Wie das Staatssekretariat für Wirtschaft (seco) auf Anfrage mitteilt, sind derzeit Bestrebungen im Gange, die Transparenz im Pariser Klub zu verbessern.

Die eidg. Räte ermächtigten den Bundesrat nach den Ratsverhandlungen im Dezember 1999 im Ständerat und im März 2000 im Nationalrat für weitere zehn Jahre, Schuldenkonsolidierungsabkommen zu vereinbaren ${ }^{41}$.

\section{$\square$ Umschuldung kommerzieller Schulden}

Im Jahre 1999 schlossen sieben Schuldnerländer Umschuldungsabkommen mit ihren kommerziellen Gläubigern, wobei 7,1 Milliarden Schulden restrukturiert und 2,7 Milliarden ausstehende Schulden reduziert wurden ${ }^{42}$.

Guyana als Land mit tiefem Einkommen konnte 34,4 Milliarden Dollar der total 55,9 Milliarden Dollar Schulden gegenüber privaten Gläubigern zu neun Prozent zurückkaufen. Die Kosten der Operation von 3,1 Milliarden Dollar trugen die Schweiz (2 Mrd. \$) und die IDA-Debt Reduction Facility.

Von den Ländern mit mittleren Einkommen konnten Argentinien, Brasilien, Mexiko, die Philippinen und Uruguay insgesamt für 6,9 Milliarden Dollar Brady-Bonds zurückkaufen. Auch die Ukraine konnte für 163 Millionen EuroBonds restrukturieren.

38. Bericht des Bundesrates zur Aussenwirtschaftspolitik 99/1+2 vom 12. Januar 2000, BBl.

39. ERG, Bericht 99, Bern/Zürich 2000.

40. Brian Caplen, Paris Club comes under attack, in: Euromoney, September 2000.

41. Botschaft des Bundesrates über den Abschluss von Schuldenkonsolidierungsabkommen vom 20. September 1999, BBl 1999, 972 lff. sowie Amtliches Bulletin, Dezember 99 (StR) und März 2000 (NR), www.parlament.ch/ab/data).

42. The World Bank, GDF 2000, aaO., Appendix 2, S. $155 \mathrm{ff}$. 


\section{$\square$ Das Schweizer Entschuldungsprogramm}

Das Schweizer Entschuldungsprogramm legt je länger, je mehr das Hauptgewicht auf multilaterale Instrumente. Der bilaterale Teil läuft langsam aus.

Von dem für Entschuldungsmassnahmen bereitgestellten 700-Jahre-Jubiläumsfonds von 400 Millionen Franken (aufgestockt durch 113 Millionen Franken vom früheren Rahmenkredit für wirtschafts- und handelspolitische Massnahmen) waren bis Mitte November 2000 insgesamt 355,5 Millionen verpflichtet. Darin ist ein weiterer Beitrag von 25 Millionen Franken an den HIPC-Trust Fund der Weltbank eingeschlossen (weitere 25 Millionen sind für das Jahr 2001 zugesagt). Somit bleibt ein Restsaldo von 44,5 Millionen Franken.

Im Jahre 2000 wurden keine neuen bilateralen Forderungen umgeschuldet. Ende November wurde ein Abkommen mit Jordanien abgeschlossen. Die Schweiz finanziert für 5 Millionen Franken Schulden von Jordanien gegenüber dem IWF und der Weltbank.

Im abgelaufenen Jahr finanzierte die Schweiz keine weiteren Rückkäufe kommerzieller Schulden mittels der IDA Debt Reduction Facility. In Vorbereitung sind Operationen mit Jemen, Kamerun und Honduras. Weitere Leistungen dürften dann keine mehr geleistet werden. Noch vorhandene Mittel sollen nach Auskünften des seco zur Finanzierung der HIPC-Initiative transferiert werden.

Die Schweiz gewährte Honduras im Juli 2000 fünf Millionen Franken zur Begleichung des Schuldendienstes gegenüber multilateralen Organisationen und für technische Hilfe bei der Schuldenverwaltung ${ }^{43}$.

Die Schweiz leistete im Jahre 2000 eine weitere Million Franken für technische Hilfe im Entschuldungsbereich. Davon wurden 0,6 Millionen Franken als allgemeiner Beitrag an das HIPC-Debt Management Capacity Program des Debt Relief International geleistet und 0,4 Millionen für regionale Programme in Zentralamerika, Westafrika und im südlichen Afrika. Die Schweiz beteiligte sich an drei Treffen auf Ministerebene mit den HIPC-Staaten, nämlich im Dezember 1999 in Kopenhagen, im Juni 2000 in Genf und im September 2000 in Prag.

\section{$\square$ NRO-Schuldenkampagnen}

Ins Zentrum ihrer alljährlichen Fastenaktion stellten die kirchlichen Hilfswerke Fastenopfer und Brot für alle ihre Kampagne für ein internationales Insolvenzrecht. Sie lancierten eine Briefaktion an die Schweizer Exekutivdirektoren bei IWF und Weltbank. Diese sollten sich für eine Art Konkursrecht für hoch verschuldete Entwicklungsländer einsetzen ${ }^{44}$. Ein Symposium «Ein Recht für Staaten auf Insolvenz?» mit internationaler Beteiligung lancierte am 9. März diese Aktion. «Brot für alle»-Präsident Christoph Eymann (NR, lib, BS) reichte in der Frühjahrssession eine entsprechende Motion ein, zusammen mit einer Gruppe mit anderen Vorstössen für eine neue internationale Finanzordnung.

Der Bundesrat erachtet die Schaffung eines internationalen Konkursgerichtes als problematisch und unzweckmässig. Damit wären nicht zumutbare Eingriffe in die Souveränität des Schuldnerlandes verbunden ${ }^{45}$.

43. seco, Pressemitteilung, 19. Juli 2000: 5 Millionen Franken für Honduras (www2.seco-admin. ch/).

44. Brot für alle/Fastenopfer, Aktionsmagazin 2000, Brot für alle/Fastenopfer (Hrsg.), Schuldenerlass durch ein Insolvenzrecht für Staaten?, Ergebnisse des Symposiums vom 9. März 2000. Entwicklungspolitische Impulse 00/1Bern 2000 und weiteres Kampagnenmaterial.

45. Amtliches Bulletin, Nationalrat, Herbstsession 2000, Neunte Sitzung, 4. Oktober 2000, Bern 2000. 
Unterdessen hat sich denn auch ein internationales NRO-Komitee gebildet, das diese Idee sowohl im Norden wie im Süden weiter verfolgen wird, und zwar unter dem Namen «Fair and Transparent Arbitration Process (FATP)».

Die Kampagne für Entschuldung und Entschädigung im südlichen Afrika, getragen von kirchlichen und kleineren Hilfswerken sowie zahlreichen Aktionsgruppen, treten in einer vielfältigen Kampagne für die Entschädigung der Apartheidsopfer und die Streichung illegitimer Schulden (odious debt) ein. Nach dem international initiierten offenen Brief von 1999 an Ruth Dreifuss, der damaligen Bundespräsidentin, unterstützte die Kampagne im Jahre 2000 zwei regionale Seminarien in Lusaka (ANROla) und Durban (Südafrika) über "apartheid-caused$d e b t$ » sowie ein weiterer internationaler Workshop in Johannesburg. Die Kampagne äusserte sich kritisch zum interdepartementalen Bericht von Ende 1999 über die Schweizer Politik gegenüber Südafrika, organisierte zahlreiche Veranstaltungen (u.a. einen Schweiz-Besuch des südafrikanischen Bischofs Ndungane im Mai 2000) und publizierte Artikel und Studien ${ }^{46}$.

\subsection{INTERNATIONALE INVESTITIONEN}

Gemäss UNCTAD sind 1999 die weltweiten Zuflüsse an ausländischer Direktinvestition (FDI) als Folge grenzüberschreitender Zusammenschlüsse und Firmenkäufe erneut stark gestiegen, nämlich von 680 auf 865 Milliarden Dollar.

\section{$\square$ Der UNCTAD-Weltinvestitionsbericht 2000}

Der weltweite Bestand ausländischer Direktinvestitionen belief sich per Ende 1999 auf 4772 Milliarden Dollar (Vorjahr: 4015 Mrd. \$). Davon wurden 3231 Milliarden in Industrieländern und 1438 in Entwicklungs- und Transitionsländern investiert ${ }^{47}$.

Von den 1999 neu investierten 865 Milliarden Dollar flossen 636 Milliarden in Industrieländer und 208 Milliarden in Entwicklungsländer (Tabelle 4). Den grössten Zufluss erreichten China (40 Mrd.), Brasilien (31 Mrd.), Argentinien (23 Mrd.), Hongkong (23 Mrd.), Mexiko (11 Mrd.) und Korea (10 Mrd.). Mit Ausnahme der Karibik und der zentralasiatischen Länder verzeichneten alle Regionen höhere Zuflüsse als im Vorjahr. Auch die afrikanischen Länder erhielten mehr Auslandsinvestitionen als im Vorjahr, doch der Hauptteil floss nach ANROla, Ägypten, Nigeria, Südafrika und Marokko. Entweder waren es Investitionen im Minen- und Erdölsektor oder es war die Folge der Privatisierung des Telekommunikationsbereiches.

Die Investitionen stammten fast ausschliesslich aus Industrieländern. Lediglich 66 der 800 Milliarden abgeflossener Direktinvestitionen stammen aus Entwicklungs- und Transitionsländern, davon 20 Milliarden aus Hongkong und 15 Milliarden aus Bermudas. Chile, Taiwan und Singapur wiesen ebenfalls bedeutende Auslandsinvestitionen aus.

46. Eine Übersicht bietet die Website der Internationalen Kampagne für Entschuldung und Entschädigung im südlichen Afrika (www.nasa-basel.ch/apartheid.html) oder von COTMEC (www.cath.ch/cotmec). Vgl. auch: AFP, Finanzplatz Aktion Schweiz: odious debt. Die Hinterlassenschaft der Diktatoren. Aus der Publikationsreihe Apartheid-Connections (2), Basel, Oktober 2000.

47. UNCTAD, World Investment Report 2000: Crossborder Mergers and Acquisitions and Development. New York und Genf, 2000. 
Tabelle 4: Ausländische Direktinvestitionen, nach Ländern und Regionen, 1997-1999, in Milliarden Dollar

\begin{tabular}{|c|c|c|c|c|c|c|}
\hline \multirow[b]{2}{*}{ Region/Land } & \multicolumn{3}{|c|}{ Zufliessende FDI } & \multicolumn{3}{|c|}{ Abfliessende FDI } \\
\hline & 1997 & 1998 & 1999 & 1997 & 1998 & 1999 \\
\hline Industrieländer & 473.0 & 680.0 & 865.0 & 404.0 & 652.0 & 732.0 \\
\hline Europa & 139.0 & 260.0 & 315.0 & 246.0 & 445.0 & 533.0 \\
\hline Schweiz & 6.6 & 7.5 & 3.4 & 17.7 & 16.6 & 17.9 \\
\hline Nordamerika & 117.0 & 208.0 & 301.0 & 122.0 & 177.0 & 168.0 \\
\hline Japan & 3.2 & 3.2 & 12.7 & 26.0 & 24.2 & 22.7 \\
\hline Entwicklungsländer & 179.0 & 179.0 & 208.0 & 64.0 & 33.0 & 66.0 \\
\hline Lateinamerika/Karibik & 69.0 & 74.0 & 90.0 & 15.0 & 9.0 & 27.0 \\
\hline Asien & 102.0 & 97.0 & 106.0 & 47.0 & 23.0 & 37.0 \\
\hline - davon Südostasien & 94.0 & 87.0 & 96.0 & 47.0 & 27.0 & 36.0 \\
\hline Osteuropa/Zentralasien & 19.0 & 20.0 & 21.0 & 3.0 & 2.0 & 3.0 \\
\hline Afrika südl. Sahara* & 4.5 & 5.2 & 6.0 & 1.2 & 0.3 & 0.6 \\
\hline Nordafrika & 2.4 & 2.3 & 3.0 & 0.4 & 0.3 & 0.3 \\
\hline Ärmste Entwicklungsländer & 2.5 & 3.7 & 4.5 & 1.0 & 0.1 & 0.4 \\
\hline Total & 473.0 & 680.0 & 865.0 & 472.0 & 687.0 & 800.0 \\
\hline
\end{tabular}

Quelle: UNCTAD, World Investment Report 2000, Table B.1/B.2, S. $283 \mathrm{ff}$

* Ohne Südafrika.

Die Differenzen im Total zwischen zu- und abfliessenden FDI ergeben sich aus unterschiedlichen statistischen Erfassungsmethoden.

Der UNCTAD-Weltinvestitionsbericht 2000 beschäftigt sich insbesondere mit den Ursachen und Auswirkungen der grenzüberschreitenden Zusammenschlüssen und Firmenkäufen (mergers and acquisitions, $M \& A$ ) und bietet dazu eine Fülle von Daten und Informationen. In kurzfristiger Sicht würden M\&A weder unbedingt neue Produktionsanlagen und Arbeitsplätze schaffen noch sei Know-how-Transfer verbunden. Sog. Greenfield-Investitionen (Investitionen in neue Anlagen) würden hinsichtlich Entwicklungswirksamkeit weit besser abschneiden. Oft würden M\&A in den Entwicklungsländern sogar die Angst schüren, die Kontrolle über wichtige wirtschaftliche Bereiche gehe ins Ausland verloren. Mit zunehmender Marktmacht sei oft sogar eine Verminderung des Wettbewerbes auszumachen. Deshalb empfiehlt die UNCTAD eine Stärkung der nationalen, regionalen und internationalen Wettbewerbspolitik. Auf längere Frist würden sich die Unterschiede zwischen M\&A und Greenfield-Investitionen allerdings vermindern.

\section{$\square$ Direktinvestitionen in und aus der Schweiz}

Der UNCTAD-Investitionsbericht 2000 weist für die Schweiz im Jahre 1999 einen Bestand von schweizerischen Investitionen im Ausland von 199 Milliarden Dollar aus, 18 Milliarden mehr als im Vorjahr. Umgekehrt belaufen sich ausländische Direktinvestitionen in der Schweiz auf 73 Milliarden Dollar (Vorjahr : 70 Mrd. \$).

Die Schweizerische Nationalbank weist für 1999 gemäss provisorischen Angaben Kapitalexporte in Schwellenländer von 6 Milliarden Franken und in andere Entwicklungsländer von 5,2 Milliarden Franken aus (Tabelle 5).

Ca JSDW 2001, Analysen und Stellungnahmen, Beitrag zu den schweizerischen Direktinvestition von Jürg Bärlocher. 
Grösste Empfängerländer schweizerischer Direktinvestitionen waren 1999 Singapur (3,6 Mrd. Fr.), Brasilien (0,8 Mrd. Fr.), Hongkong (0,6 Mrd. Fr.) und Mexiko (0,5 Mrd. Fr.). Direktinvestitionen aus Schwellenländern in der Schweiz nahmen geringfügig um 11 Millionen Franken ab, und aus andern Entwicklungsländern stiegen sie um 158 Millionen Franken.

Tabelle 5: Schweizerische Direktinvestitionen in Entwicklungs- und Schwellenländern, 1997-1999 (in Mio. Fr.)

\begin{tabular}{lrrrrrr}
\hline \multirow{2}{*}{ Region } & \multicolumn{3}{c}{ FDI Kapitalexport } & \multicolumn{3}{c}{ FDI Bestand } \\
& $\mathbf{1 9 9 7}$ & $\mathbf{1 9 9 8}$ & $\mathbf{1 9 9 9}$ & $\mathbf{1 9 9 7}$ & $\mathbf{1 9 9 8}$ & $\mathbf{1 9 9 9}^{\circ}$ \\
\hline Schwellenländer & 2176 & 5203 & 6042 & 21822 & 24682 & 31658 \\
\hline Lateinamerika & -207 & 1243 & 1266 & 8894 & 8908 & 10972 \\
\hline Asien & 2383 & 3690 & 4776 & 12929 & 15774 & 20685 \\
\hline Entwicklungsländer & 1793 & 2752 & 5240 & 28956 & 28902 & 36286 \\
\hline Lateinamerika & 935 & 2209 & 4876 & 23665 & 23892 & 29567 \\
\hline Afrika & 230 & 102 & 295 & 1607 & 1611 & 2616 \\
\hline Asien & 629 & 441 & 69 & 3684 & 3400 & 4103 \\
\hline Industrieländer & 21765 & 16153 & 40574 & 189877 & 196300 & 242202 \\
\hline Alle Länder & 25734 & 24107 & 51856 & 240655 & 249884 & 310146 \\
\hline
\end{tabular}

Quelle: Schweizerische Nationalbank, Zahlungsbilanz der Schweiz 1999, SNB Zürich, September 2000.

${ }^{\mathrm{P}}$ provisorisch.

\section{$\square$ Bilaterale und multilaterale Investitionsverträge}

Die Zahl der bilateralen Investitionsverträge zwischen einzelnen Staaten wuchs weiter an. Ende 1999 waren 1856 solcher Verträge in Kraft. Von den 130 im Jahre 1999 in Kraft getretenen Abkommen wurden fast die Hälfte zwischen Entwicklungsländern untereinander abgeschlossen, während knapp ein Drittel auf Abkommen zwischen Entwicklungs- und Industrieländern fallen ${ }^{48}$. Dazu kommen weitere Verträge mit regionaler Tragweite, die auch Investitionsregeln beinhalten.

Die OECD setzte im Juni 2000 Richtlinien für multinationale Unternehmungen in Kraft mit Empfehlungen für Arbeits- und Umweltstandards, zur Bekämpfung der Korruption und zum Schutz von Konsumentenrechten ${ }^{49}$.

DSDW 2001, Analysen und Stellungnahmen, Bastienne Joerchel Anhorn, Die OECD-Leitsätze für Multinationale Unternehmen.

\subsection{DIE INTERNATIONALEN FINANZINSTITUTIONEN}

Die Bretton-Woods-Institutionen sahen sich einem starken und kontroversen Reformdruck ausgesetzt. Die Armutsbekämpfung und das Schuldenproblem der ärmsten Länder standen im Vordergrund. Eine klarere Rollentrennung, aber auch eine verstärkte Zusammenarbeit zwischen IWF und Weltbank wurde festgelegt.

48. UNCTAD, World Investment Report 2000, aaO., S. 6 und 8.

49. OECD, News Release, New Guidelines for Multinational Enterprises reinforce framework for the global economy, Paris, 27. Juni 2000 (www.oecd.org/media/release/nw00-68a.htm). 


\subsubsection{DER INTERNATIONALE WÄHRUNGSFONDS (IWF)}

Der Rücktritt des Geschäftsführenden Direktors, die Wahl seines Nachfolgers, die Reform der Kreditinstrumente, die Verbesserung der Überwachungstätigkeit sowie eine Reihe institutioneller Neuerungen waren die herausragenden Ereignisse im IWF.

\section{$\square$ Rückläufige Kreditzusagen}

Nach den rekordhohen Kreditvergaben der vergangenen zwei Geschäftsjahre sanken die Kreditzusagen auf 22,9 Milliarden Sonderziehungsrechte (SZR, 1 SZR entspricht 1,31921 US \$). Auch die Kreditauszahlungen sanken auf knapp einen Drittel der vorangegangen Geschäftsjahre und belief sich im Geschäftsjahr 1999/2000 noch auf 6,8 Milliarden SZR ${ }^{50}$. Das ausstehende Kreditvolumen fiel demzufolge auf 50,4 Milliarden SZR gegenüber 67,2 Milliarden im Vorjahr. Es wurden für rund 17 Milliarden SZR mehr Kredite zurückbezahlt, als neue ausbezahlt wurden. Dadurch stieg die Liquiditätsquote wieder auf 153 Prozent und näherte sich dem Wert vor der Asienkrise (vgl. Tabelle 6).

Der IWF genehmigte elf neue Bereitschaftskreditvereinbarungen, vier erweiterte Kreditvereinbarungen und zehn Vereinbarungen im Rahmen der Armutsreduzierung- und Bekämpfungsfazilität (Poverty Reduction and Growth Facility, $P R G F$ ). Die Anzahl der Kreditverträge blieb mit 58 praktisch konstant (Stand Vorjahr: 56).

Tabelle 6: Kreditvergabe des IWF, 1996 - 2000 (in Mrd. SZR, jeweilen per 30.4.)

\begin{tabular}{lrrrrr}
\hline & $\mathbf{1 9 9 6}$ & $\mathbf{1 9 9 7}$ & $\mathbf{1 9 9 8}$ & $\mathbf{1 9 9 9}$ & $\mathbf{2 0 0 0}$ \\
\hline Kreditzusagen & 19.9 & 5.3 & 32.2 & 29.4 & 22.9 \\
\hline Kreditauszahlungen & 12.3 & 5.6 & 19.9 & 22.2 & 6.8 \\
\hline Rückzahlungen & 7.1 & 7.2 & 4.4 & 11.1 & 23.6 \\
\hline Nettotransfer & 5.2 & -1.6 & 15.5 & 11.1 & -16.8 \\
\hline Ausstehende Kredite & 42.0 & 40.5 & 56.0 & 67.2 & 50.4 \\
\hline Überfällige Verpflichtungen & 2.2 & 2.2 & 2.3 & 2.3 & 2.3 \\
\hline Zahl der Kreditverträge & 57.0 & 60.0 & 60.0 & 56.0 & 58.0 \\
\hline
\end{tabular}

Quelle: IMF, Jahresbericht 2000.

\section{$\square$ Reform der Kreditinstrumente}

Damit der IWF seine Rolle im internationalen Finanzsystem besser wahrnehmen kann, straffte er seine Kreditinstrumente. Vier überholte Fazilitäten wurden abgeschafft $^{51}$. Mit Reformmassnahmen will der IWF künftig verhindern, dass die Mitgliedsländer mehrfach hintereinander IWF-Kredite beanspruchen und damit den IWF zu einem langfristigen Kreditgeber machen. Die Schweiz lehnte allerdings mit andern europäischen Ländern die Vorschläge der G-7-Länder ab, strikte unterschiedliche Zinssätze je nach Kreditdauer anzuwenden (Zinsgraduierung) ${ }^{52}$. Weil die vor Jahresfrist neu eingeführten Contingent Credit Lines $(C C L)$ nie verwendet worden waren, modifizierte der IWF auch dieses Kreditinstrument.

50. IMF, Jahresbericht 2000: Die Vorteile der Weltwirtschaft für alle sichern. Washington, 2000.

51. IMF, Jahresbericht 2000, aaO. Kasten 6.1: Finanzfazilitäten und Politikmassnahmen des IWF, S. $74 / 75$.

52. IMF, Executive Director, Activity Report 2000, aaO., S. 7. 
Der IWF musste auch Massnahmen ergreifen gegen verfälschende oder irreführende Informationen von Mitgliedsländer sowie missbräuchliche Verwendung von Krediten. Im Falle von Russland verdichteten sich vorerst Hinweise, dass IWF-Gelder, die 1998 ausbezahlt worden waren, in der Schweiz gelandet sein könnten. Entsprechende Untersuchungen brachten allerdings nichts Konkretes zutage. Im Falle der Ukraine führte die Zentralbank Transaktionen durch, um den wirklichen Stand der Währungsreserven zu beschönigen. Die Credit Suisse First Bosten war darin involviert, was NR Neirynck (CVP, VD) veranlasste, in der Fragestunde des Nationalrates $\mathrm{zu}$ intervenieren ${ }^{53}$. Die Eidg. Bankenkommission untersuchte die Rolle der Credit Suisse First Boston.

In Zusammenhang mit den Vorbereitungen für die zwölfte Quotenrevision drängten die USA auf eine bessere Vertretung der Schwellenländer und auf einen Abbau der europäischen Übervertretung. Weil damit auch der Sitz der Schweiz im Exekutivrat gefährdet sein könnte, setzte Bundesrat Kaspar Villiger eine interdepartementale Arbeitsgruppe ein, die untersuchen soll, wie der Schweizer Sitz am besten zu verteidigen sei. Ein Bericht ist auf Anfang 2001 zu erwarten. In diesem Zusammenhang ist eine weitere Verbesserung der Zusammenarbeit mit den andern Ländern der Stimmrechtsgruppe zu sehen. Bundesrat Kaspar Villiger unternahm Ende Oktober/Anfang November eine Reise in die zentralasiatischen Länder der Stimmrechtsgruppe ${ }^{54}$.

\section{$\square$ Institutionelle Reformen}

Der IWF hält nicht nur seine Mitgliedsländer zu verbesserter Transparenz an. Vielmehr führte er im abgelaufenen Jahr eine Reihe interner Reformen durch. Er verbesserte den Zugang der Öffentlichkeit zum IWF-Archiv, gibt nun mehr Informationen über seine finanzielle Situation, publiziert eine Zusammenfassung des Arbeitsprogramms des Exekutivrates und überprüfte die bisherige Informationspolitik. Der IWF veröffentlicht heute einen Grossteil seiner Papiere auf dem Internet ${ }^{55}$. Die Schweiz unterstützt die Politik verbesserter Transparenz.

Die Schweiz unterstützte ebenfalls die Schaffung eines unabhängigen Evaluationsbüros (Independent Evaluation Office, EVO). Externe Evaluationen sind weiterhin möglich. So wurde im März 2000 die Evaluation der Forschungstätigkeit abgeschlossen, deren Resultate vollumfänglich auf der IMF-Website veröffentlicht wurden.

\section{$\square$ Neuer geschäftsführender Direktor}

Im Februar 2000 trat Michel Camdessus nach 13 Dienstjahren als geschäftsführender Direktor zurück. Am 23. März 2000 ernannte der Exekutivrat nach langwierigem Hin und Her den Deutschen Horst Köhler zu seinem Nachfolger. Das wenig transparente Verfahren und der fixe Anspruch Europas auf den IWFVorsitz wurde weltweit kritisiert. Die Arbeitsgemeinschaft der Hilfswerke unterstützte einen von zahlreichen internationalen NRO unterzeichneten Brief, in dem transparentere Verfahren gefordert wurden. IWF und Weltbank haben nun je eine Arbeitsgruppe eingesetzt, die den Auswahlprozess für hohe Posten überprüfen

53. Amtliches Bulletin, Frühjahrssession, NR, 20. März 2000, Frage 00.5074 (www.parlament.ch/).

54. EFD-Medienmitteilung 27. Oktober 2000: Reise von Bundesrat Villiger nach Zentralasien und dem Kaukasus (www.efd.admin.ch/dok/presse/mm1000/zentralasien.htm).

55. Die täglich aktualisierte Website des IWF ist abrufbar unter: www.imf.org. 
soll. Bei der Weltbank wird diese Arbeitsgruppe vom Schweizer Exekutivdirektor Matthias Meyer geleitet.

Im Jahre 1999 hatte der Nationalrat der parlamentarischen Initiative Gysin zur Genehmigung von Kapitalaufstockungen des IWF durch das Parlament knapp Folge gegeben. Der Bericht der Aussenpolischen Kommission des Nationalrates wurde mittlerweile im Bundesblatt publiziert ${ }^{56}$. Die Stellungnahme des Bundesrates, Diskussion und Beschluss stehen noch aus.

\subsubsection{DIE WELTBANK}

Die Weltbankgruppe (IBRD und IDA) hat im abgelaufenen Geschäftsjahr insgesamt bloss noch halb so viel Kredite zugesagt wie im Krisenjahr zuvor. Auch die Auszahlungen sanken, weniger stark bei der IDA als bei der IBRD (vgl. Tabelle 7). Die rekordhohen Ausleihungen in den beiden Vorjahren waren bedingt durch die Finanzkrise. Die Qualität der finanzierten Projekte sank weiter. Der Anteil der Projekte mit unsicherer Zielerfüllung sank auf 15 Prozent. Die Weltbank nahm in 13 Währungen insgesamt 15,8 Milliarden Dollar auf den Kapitalmärkten auf, darunter erstmals im Januar 2000 eine elektronisch aufgelegte Bondanleihe von 3 Milliarden Dollar ${ }^{57}$.

Die Ausleihungen (IBRD und IDA) flossen zu 26 Prozent nach Lateinamerika und in die Karibik, zu je 20 Prozent nach Osteuropa/Zentralasien und nach Ostasien/Pazifik, zu je 14 Prozent nach Afrika und Südasien sowie zu 6 Prozent nach Nordafrika/Mittlerer Osten.

Tabelle 7: Ressourcenfluss der Weltbank 1997-2000 (in Mrd. \$, jeweilen per 30.6.)

\begin{tabular}{lrrrr}
\hline & $\mathbf{1 9 9 7}$ & $\mathbf{1 9 9 8}$ & $\mathbf{1 9 9 9}$ & $\mathbf{2 0 0 0}$ \\
\hline Weltbank & & & & \\
\hline Kreditzusagen & 14.5 & 21.1 & 22.2 & 10.9 \\
\hline Auszahlungen & 14.0 & 19.2 & 18.1 & 13.3 \\
\hline Ausstehende Kredite & 105.8 & 106.6 & 117.2 & 120.1 \\
\hline IDA & & & & \\
\hline Kreditzusagen & 4.6 & 7.5 & 6.8 & 4.4 \\
\hline Auszahlungen & 6.0 & 5.6 & 6.0 & 5.2 \\
\hline Ausstehende Kredite & & 78.4 & 83.7 & 86.6 \\
\hline
\end{tabular}

Quelle: The World Bank, Annual Report 2000, und mündliche Angaben der Weltbank.

Die Bank fokussierte ihre Tätigkeit noch stärker auf das erklärte Hauptziel, die Armutsbekämpfung. Sie unterstützte dabei aber auch marktfreundliche Politiken und Institutionen. Sie baute ihr Kreditinstrumentarium und ihr Dienstleistungsangebot aus.

Der schweizerische Exekutivdirektor charakterisierte das vergangene Geschäftsjahr mit zwei Wörtern: Innovation and Controversy ${ }^{58}$. Als innovativ bezeichnete Matthias Meyer die Armutsbekämpfung mit den zentralen Elementen der Poverty Reduction Strategy Papers (zusammen mit dem IWF) und dem Com-

56. Parlamentarische Initiative, Genehmigung von Kapitalaufstockungen des IWF durch das Parlament. Bericht der Aussenpolitischen Kommission des Nationalrates vom 15. Mai 2000, in: BB1 2000, 4031.

57. The World Bank, Annual Report 2000, Washington, 2000.

58. The World Bank, Executive Director, Report to the Governors, aaO. 
prehensive Development Framework (CDF), ferner die enge Zusammenarbeit zwischen der Bank und ihrer Tochter IFC in der Förderung des privaten Sektors, die Zuwendung zu Fragen globaler öffentlicher Güter, der Reform wichtiger öffentlicher Institutionen sowie der Korruptionsbekämpfung.

JSDW 2000, zu CDF vgl. Der institutionelle Umbau, S. 145.

Der diesjährige World Development Report 2000/2001 ist der Armutsbekämpfung gewidmet ${ }^{59}$. Er entstand nach einer breit angelegten mehrjährigen Konsultation, der auch zu Kontroversen führte. Der Teamleiter Ravi Kanbur trat nach ideologischen Kontroversen mit dem US-Schatzminister Summers zurück. Der Bericht legt das Gewicht auf drei Stossrichtungen: Chancengleichheit (Opportunity), Ermächtigung (Empowerment) und Sicherheit. Vor allem die Ermächtigung stiess bei orthodoxen Ökonomen auf Widerstand60. Der Bericht postuliert aber auch Massnahmen auf globaler Ebene. Zum Beispiel fordert die Weltbank einen besseren Zugang der Entwicklungsländer zu den Märkten in den Industrieländern.

Heftige Kontroversen löste die Bank auch aus bei zwei grossen Projekten. Sie verabschiedete die Mitfinanzierung des Tschad-Kamerun-Pipeline-Projektes, aber mit unterschiedlich bewerteten Auflagen sowie einer laufenden internationalen Überwachung. Diese zusätzlichen Auflagen waren die Folge einer breiten internationalen Kampagne. Die Schweiz stimmte ebenfalls zu, nachdem sie im Laufe des Verfahrens zwar stets eine positive, aber kritische Haltung eingebracht hatte.

Bei einem Umsiedelungsprojekt in China in mehrheitlich von tibetanischen Angehörigen bewohnten Gebiet führte die öffentliche Kontroverse zu zusätzlichen Untersuchungen und Auflagen, die China letztlich bewog, das Projekt von der Finanzierung durch die Weltbank zurückzuziehen. Die Schweiz hätte dem Projekt trotz Opposition vor allem seitens der in der Schweiz lebenden Tibetanerinen und Tibetaner und der sie unterstützenden Bewegung zugestimmt.

\subsubsection{DIE BRETTON-WOODS-INSTITUTIONEN UNTER REFORMDRUCK}

Die Finanzkrise führte zu einer heftigen und sehr kontroversen Debatte über die Rolle von IWF, Weltbank und weiteren internationalen Institutionen. Sie wurden mehr denn je zu einem die Globalisierung symbolisierenden Objekt.

Hohe Wellen warf im März 2000 der sog. Meltzer-Report ${ }^{61}$. Im November 1998 wurde eine elfköpfige Kommission unter dem Vorsitz des den Republikanern nahe stehenden Wirtschaftsprofessors Allan Meltzer vom US-Kongress beauftragt, Empfehlungen für die künftige Rolle von IWF und Weltbank zu erarbeiten. Der Bericht schlägt vor, der IWF solle sich nur noch auf Bewältigung von Finanzkrisen mit kurzfristigen Krediten zu Strafzinsen beschränken. Die Weltbank soll die Aufgabe erfüllen, langfristige Entwicklungsfinanzierung zu leisten, aber nur noch in den 80 bis 90 ärmsten Ländern, die keinen Zugang zu den pri-

59. The World Bank, Attacking Poverty, World Development Report 2000/2001, Washington 2000.

60. NZZ, Rezepte zur Armutsbekämpfung (13. September 2000).

61. Report of the International Financial Institution Advisory Commission, Allan H. Meltzer, Chairman, 8. März 2000, Submitted to the U.S. Congress and U.S. Department of the Treasury (http://phantomx.gsiu.cmu.edu/IFIAC). 
vaten Kapitalmärkten besitzen. Dabei soll sie statt Kredite Geschenke erteilen. Den ärmsten Ländern sollten zudem die Schulden gegenüber IWF und Weltbank erlassen werden. Drei Kommissionsmitglieder verfassten einen Minderheitsgegenbericht.

Seither sind zahlreiche Stellungnahmen und Papiere von Regierungen, Wissenschaft, Banken, Medien, NRO und auch von IWF und Weltbank selber verfasst und teilweise veröffentlicht worden, nicht ausschliesslich als Antwort auf den Meltzer-Bericht, sondern vielmehr als Teil einer Debatte zur Grundsatzfrage nach der Rolle und Funktion von IWF, Weltbank und andern internationalen Organisationen ${ }^{62}$. In der Schweiz beschäftigten sich die betroffenen Verwaltungsabteilungen mit dem Meltzer-Report. Die DEZA führte mit dem Chef der Weltbank-Evaluationsabteilung einen Workshop durch, zu dem auch Vertreter von NRO eingeladen waren. Auch die Bretton-Woods-Subkommission diskutierte an einer ihrer Sitzungen Forderungen und Konsequenzen des Berichtes.

Der Meltzer-Bericht hat zweifellos mit beigetragen, die Reformen in IWF und Weltbank voranzutreiben. Zudem ging der neue geschäftsführende Direktor des IWF, Horst Köhler, mit einer bemerkenswerten Offenheit an seine Aufgabe. Köhler und Weltbank-Präsident James Wolfensohn veröffentlichten kurz vor der Jahresversammlung in Prag im September 2000 ein gemeinsames Papier, in dem sie die Ziele, Aufgaben, die Arbeitsteilung und die Zusammenarbeit der beiden Institutionen darlegten 63 . Ziel beider Institutionen sei es, den Ländern bei ihren selbst erarbeiteten Strategien zu helfen, die Lebensqualität zu fördern sowie die Armut durch nachhaltiges und ausgeglichenes Wachstum für alle zu reduzieren. Dem IWF komme dabei die Aufgabe zu, die finanzielle und makroökonomische Stabilität zu fördern, während die Bank sich auf strukturelle, institutionelle und soziale Dimensionen der Entwicklung konzentrieren soll. Bank und Fonds sollen eng zusammenarbeiten. Köhler und Wolfensohn riefen die reichen Länder aber auch dazu auf, ihre Märkte zu öffnen, die öffentliche Hilfe zu steigern und den ärmsten Ländern die Schulden zu erlassen.

Die Kontroverse berührte aber nicht bloss institutionelle Reformen, sondern ebenso die politische Ausrichtung von IWF und Weltbank. Bisher galt der so genannte Washington-Konsens als Massstab für die politische Richtung. Danach wären für nachhaltiges Wachstum eine ausgeglichene makroökonomische Politik notwendig. Zu dessen Elementen gehören eine ausgeglichene Zahlungsbilanz, eine tiefe Inflation, ein ausgeglichenes Staatsbudget, eine tiefe Staatsquote, eine Förderung des privaten Sektors, ein exportorientierter, liberaler Aussenhandel, die Privatisierung von Staatsbetrieben.

Die neue ideologische Ausrichtung verlangt eine mehr institutionell ausgerichtete Politik und eine breit abgestuitzte Wachstumspolitik. Einer der markantesten und provozierenden Vertreter dieser Richtung, Joe Stiglitz, trat schliesslich unter Protest als Chefökonom der Weltbank zurück. Neben Wachstum wurde auch die Verteilung des Einkommens wieder zu einem Thema. Beigetragen zur Kontro-

62. Stellvertretend für die reiche Literatur: The Heinrich Böll Foundation, Washington Office, A Makeover for the Bretton Woods Twins? A Transatlantic Critique of the Meltzer Report and Other Reform Proposals. Washington 2000 (www.boell.org).

63. IMF/The World Bank Group: An Enhanced Partnership for Sustainable Growth and Poverty Reduction. Joint Statement by Horst Köhler and James Wolfensohn, Washington, 5. September 2000 (www.imf.org/external/np/omd/2000/part.htm). 
verse hat auch eine Studie von Weltbank-Mitarbeitern über die Bedeutung von Wachstum und die Funktion des Wachstums für die Armutsbekämpfung64. Wie qualitatives Wachstum zu erreichen ist, und wie es für alle nutzbar ist, war das herausforderndste Thema der wirtschaftspolitischen Debatte in und um IWF und Weltbank 65 .

\subsubsection{DIE JAHRESTAGUNG VON IWF UND WELTBANK}

Sowohl das Frühjahrestreffen von IWF und Weltbank im April in Washington wie auch die Jahresversammlung im September in Prag erreichten eine höhere Aufmerksamkeit der Medien, verglichen mit den früheren Treffen. Hauptgrund war, dass zahlreiche Organisationen diese Versammlungen wie jene weiterer internationaler Organisationen zum Anlass zu Demonstrationen gegen die Globalisierung nahmen.

Die weltweite Mobilisierung gegen die Nachteile der Globalisierung forderte aber auch die Bretton-Woods-Institutionen zu Reaktionen. Armutsbekämpfung und Verteilung der Früchte des Wachstums für alle war das dominierende Thema sowohl in Washington wie in Prag. Zurück bleibt die Frage, ob sich bloss die Rhetorik oder auch die Praxis geändert haben.

Thematisch beschäftigte sich der Internationale Währungs- und Finanzausschuss am Frühjahrestreffen im April mit der Reform der IWF-Kreditinstrumente, der Verbesserung der internationalen Finanzarchitektur und verabschiedete Michel Camdessus. Der Entwicklungsausschuss genehmigte die Weltbankstrategie zur Bekämpfung von HIV/AIDS. Beide Ausschüsse überprüften die Fortschritte bei der HIPC-Initiative und der Armutsbekämpfungsstrategie.

Die Jahresversammlung in Prag im September stand im Zeichen der Reformen von IWF und Weltbank. Im Mittelpunkt der Beratungen des Internationalen Währungs- und Finanzausschusses standen die Vorschläge für die neue Fokussierung des IWF, die Reform der IWF-Kreditinstrumente, die Stärkung des Internationalen Finanzsystems sowie die Auswirkungen der Erdölpreissteigerungen auf den Gang der Weltwirtschaft ${ }^{66}$. Der Entwicklungsausschuss prüfte die Weltbankstrategien für globale, grenzüberschreitende Probleme und diskutierte besser angepasste Strategien und Instrumente für Entwicklungsländer mit unterschiedlichem Einkommen. Gemeinsam bestätigten der Entwicklungs- und der Währungsausschuss die Wichtigkeit weiterer Fortschritte in der Entschuldung der ärmsten Länder. Wie bereits letztes Jahr wurden alle wesentlichen Dokumente der Jahresversammlungen und des Frühjahrestreffens auf den Websites von IWF und Weltbank publiziert ${ }^{67}$.

Bundesrat Kaspar Villiger führte im Jahr 2000 den Vorsitz der G-10. Sein Nachfolger wird der deutsche Bundesfinanzminister Hans Eichel.

64. David Dollar and Aart Kraay, Growth is Good for the Poor, World Bank Working Papers, Washington, März 2000 (www.worldbank.org/research/growth/absddolakray.htm).

65. Ein grundlegendes Werk zu dieser Debatte ist auch: The World Bank, The Quality of Growth, Published for the World Bank by Oxford University Press, Washington/New York 2000.

66. Der World Economic Outlook vom September 2000 des IWF widmet einen Grossteil seiner Ausführungen den Erfahrungen des Transitionsprozesses sowie der Herausforderung der Integration dieser Länder in die EU: IMF, World Economic Outlook, Washington, September 2000.

67. www.imf.org und www.worldbank.org. 
Prag und Washington standen nicht nur im Zeichen der Demonstrationen, sondern auch im vertieften Dialog zwischen NRO und den Bretton-Woods-Institutionen. Während beiden Veranstaltungen fanden zahlreiche Workshops und Seminarien zwischen NRO und den Bretton-Woods-Institutionen statt.

\section{QUELLEN}

IMF :

IMF, Die Vorteile der Weltwirtschaft für alle sichern. Jahresbericht 2000, Washington 2000.

IMF Fact Sheet: A Brief Guide to Committees and Group, 17. Juli 2000. (www.imf.org/external/np/ exr/facts/groups.htm).

IMF, International Capital Markets. Developments, Prospects, and Key Policy Issues. Washington, September 2000.

IMF, Progress Report on the Bank-Fund Financial Sector Liaison Committee, 29. März 2000 (www.imf.org/external/np/mae/fslc/032900.htm).

IMF, Exchange Rate Regimes in an Increasingly Integrated World Economy, Issue Briefs for 2000, Juni 2000 (www.imf.org/external/np/exr/ib/2000/062600.HTM).

IMF, Capital Controls: Country Experiences with their Use and Liberalization. Occasional Paper 190, Washington 2000.

IMF, Involving the Private Sector in the Resolution of Financial Crisis - Standstills - Preliminary Considerations. 5. September 2000 (www.imf.org/external/np/pdr/sstill/2000/eng).

IMF, Jahresbericht 2000: Die Vorteile der Weltwirtschaft für alle sichern. Washington 2000.

IMF, Executive Director for Azerbaijan, Kyrgyz Republic, Poland, Switzerland, Tajikistan, Turkmenistan, Uzbekistan, Activity Report 2000.

IMF/World Bank, HIPC-Initiative, Country Implementation Status Notes, 19. September 2000.

IMF/World Bank, The Enhanced Initiative for Heavily Indebted Poor Countries. Review of Implementation, 7. September 2000 (www.worldbank.org/html/extdr/hipc/hippc090700.htm)

IMF/World Bank, Poverty Reduction Strategy Papers - Progress in Implementation, 7. Septbember 2000 (www.imf.org/external/np/prsp/2000/prsp.htm).

IMF/The World Bank Group: An Enhanced Partnership for Sustainable Growth and Poverty Reduction. Joint Statement by Horst Köhler and James Wolfensohn, Washington, 5. September 2000 (www.imf.org/ external/np/omd/2000/part.htm).

IMF, World Economic Outlook, Washington, September 2000.

\section{THE WORLD BANK:}

The World Bank, Global Development Finance 2000 (GDF), 2 Vol. Washington 2000.

World Bank Group, Executive Director for Azerbaijan, Kyrgyz Republic, Poland, Switzerland, Tajikistan, Turkmenistan, Uzbekistan, Report to the Governors, September 2000.

The World Bank, Annual Report 2000, Washington 2000.

The World Bank, Attacking Poverty, World Development Report 2000/2001, Washington 2000.

David Dollar and Aart Kraay, Growth is Good for the Poor, World Bank Working Papers, Washington, März 2000 (www.worldbank.org/research/growth/absddolakray.htm).

The World Bank, The Quality of Growth, Published for the World Bank by Oxford University Press, Washington/New York 2000.

\section{Bank für Internationalen Zahlungsausgleich (BIZ):}

Bank für Internationalen Zahlungsausgleich (BIZ), 70. Jahresbericht, 1. April 1999 bis 31. März 2000, Basel 2000.

BIS Press Release, Financial Stability Forum, 26. Mai 2000 (www.bis.org/press/p00526.htm).

\section{OECD:}

OECD, News Release. Attachment 2 from Press Statement by the DAC Chairman, DAC High Level Meeting, 11.-12. Mai 2000, Paris, 12. Mai 2000.

OECD, The DAC Journal. Development Cooperation 1999 Report, OECD, Paris 2000.

OECD, News Release, New Guidelines for Multinational Enterprises reinforce framework for the global economy, Paris, 27. Juni 2000 (www.oecd.org/media/release/nw00-68a.htm). 
UNO :

UNO, Financial Flows to Developing and Transition Economies and Main Policy Developments. An Excerpt from World Economic and Social Survey, 2000. Background information for the Preparatory Committee for the High-level Intergovernemental Event on Financing for Development, First Sustantive Session, 31. Mai-2. Juni 2000 (www.un.org/esa/analysis/ffd).

UNCTAD, Trade and Development Report 2000, New York und Genf 2000.

UNCTAD, The Least Developed Countries 2000 Report, Aid, Private Capital Flows and External Debt: The Challenge of Financing Development in LDCs, New York und Genf 2000.

UNCTAD, World Investment Report 2000 : Crossborder Mergers and Acquisitions and Development. New York und Genf 2000.

\section{Offizielle schweizerische Publikationen:}

Villiger Kaspar, Bundesrat, Statement an der Pressekonferenz im Anschluss an die Tagung der Zehnergruppe und des Internationalen Währungs- und Finanzausschusses. Jahrestagung der Bretton-WoodsInstitutionen 2000 (Vervielfältigung EFV, ohne Datum).

Info plus, Newsletter des EFD Reformen im IWF: Diskussion beginnt erst, Nr. 9, März 2000.

ERG, Bericht 99, Bern/Zürich 2000 (www.swiss-erg.com).

Jacques de Watteville, Das Bankgeheimnis. Das Schweizer Bankgeheimnis angesichts der jüngsten internationalen Entwicklungen, namentlich in der OECD und der $E U$, in: Die Volkswirtschaft - Magazin für WirtschaftsPolitik, 7/2000, Bern.

Botschaft des Bundesrates über den Abschluss von Schuldenkonsolidierungsabkommen vom 20. September 1999, BB1 1999.

seco-Pressemitteilung, 19. Juli 2000: 5 Millionen für Honduras (www2.seco-admin. ch/)

Amtliches Bulletin, Diverse Ausgaben National und Ständerat, Bern, (www.parlament.ch/frameset/d/ index. htm).

Bericht des Bundesrates zur Aussenwirtschaftspolitik 99/1+2 vom 12. Januar 2000, BBl.

Schweizerische Nationalbank, Zahlungsbilanz der Schweiz 1999, SNB Zürich, September 2000.

EFD-Medienmitteilung 27. Oktober 2000: Reise von Bundesrat Villiger nach Zentralasien und dem Kaukasus (www.efd.admin.ch/dok/presse/mm1000/zentralasien.htm).

Parlamentarische Initiative Genehmigung von Kapitalaufstockungen des IWF durch das Parlament. Bericht der Aussenpolitischen Kommission des Nationalrates vom 15. Mai 2000, in: BB1 2000, 4031.

NRO:

WEED, Spekulieren, Regulieren. Kapital braucht Kontrolle. Die internationalen Finanzmärkte: Funktionsweise-Hintergründe-Alternativen, Bonn, August 2000.

Swiss Coalition News, Nr. 22, Januar 2000, Bern, Re-regulation of International Financial Markets.

Arbeitsgemeinschaft Swissaid/Fastenopfer/Brot für alle/Helvetas/Caritas, Mit «civil Society " die Armut bekämpfen? Fotoprotokoll, Seminar vom 10. Mai 2000, Bern.

Brot für alle/Fastenopfer, Aktionsmagazin 2000, Bern/Luzern 2000.

AFP, Finanzplatz Aktion Schweiz: Odious Debt. Die Hinterlassenschaft der Diktatoren. Aus der Publikationsreihe Apartheid-Connections (2), Basel, Oktober 2000.

\section{Andere Publikationen :}

Institute of International Finance, Capital Flows to Emerging Market Economies, Washington, 23. September 2000 (www.iif.com).

Barry Eichengreen, Can the Moral Hazard caused by IMF-bailouts be reduced?, ICBM/CEPR, Genf/London 2000.

Joerchel Anhorn, Bastienne, Die OECD-Leitsätze für multinationale Unternehmen - ISDW, 2001.

Reform der internationalen Finanzordnung. Medienkonferenz von acht Parlamentariern vom 23. März 2000, Vervielfältigung, Bern, ohne Datum (erhältlich beim Autor dieser Übersicht).

Report of the International Financial Institution Advisory Commission, Allan H. Meltzer, Chairman, 8. März 2000, Submitted to the U.S. Congress and U.S. Department of the Treasury (http://phantomx.gsiu.cmu.edu/IFIAC).

The Heinrich Böll Foundation, Washington Office, A Makeover for the Bretton Woods Twins? A Transatlantic Critique of the Meltzer Report and Other Reform Proposals. Washington 2000 (www.boell.org). 


\section{Medien :}

Gurtner, Bruno, Geld denkt nicht. Die Verantwortlichen der internationalen Finanzmärkte haben ihre Versprechen nicht eingehalten. Cash, 29. September 2000.

Brian Caplen, Paris Club comes under attack, in: Euromoney, September 2000.

NZZ, Rezepte zur Armutsbekämpfung (13. September 2000). 